\title{
Comentáa
}

\section{Associação do Fator de Impacto e do Índice $h$ para a avaliação de periódicos científicos: uma aplicação no campo da Ciência da Informação}

\author{
Deise Deolindo Silva \\ Doutora, Universidade Estadual Paulista, Marília, SP, Brasil; \\ deisedeolindo@hotmail.com \\ Cátia Cândida de Almeida \\ Doutoranda, Universidade Estadual Paulista, Marília, SP, Brasil; \\ caticandida@gmail.com \\ Maria Cláudia Cabrini Grácio \\ Doutora, Universidade Estadual Paulista, Marília, SP, Brasil; \\ cabrini@marilia.unesp.br
}

\begin{abstract}
Resumo: Os indicadores bibliométricos oferecem relevante subsídio ao processo avaliativo da produção científica, especialmente em nível meso e macro, e para as tomadas de decisões em políticas científicas. Dentre eles, destacam-se o Fator de Impacto e o Índice $h$. Criado para a avaliação de impacto de periódicos científicos, o Fator de Impacto é definido como a média de citações recebidas por um periódico científico em um determinado período. $\mathrm{O}$ Índice $h$ considera em sua formulação aspectos relativos ao impacto científico (citação) e à produtividade (número de artigos produzidos) de um pesquisador. Diante da importância contemporânea desses indicadores, esta pesquisa objetiva propor uma forma de avaliação de periódicos científicos, combinando o Fator de Impacto e o Índice $h$, a fim de contribuir para a discussão da necessidade de uma avaliação multidimensional nos processos avaliativos. Levantaram-se os valores do Fator de Impacto e do Índice $h$ dos 168 periódicos científicos consignados à Ciência da Informação, indexados na base de dados Scopus durante os anos 2012, 2013, 2014 e coletados por meio do portal SCImago Journal \& Country Rank. Entre os resultados, observou-se que, embora haja uma tendência de posicionamento similar quanto a esses indicadores, para uma parcela significativa de periódicos as tendências são discordantes. Conclui-se que a utilização conjunta dos indicadores Fator de Impacto e Índice $h$ contribui para uma avaliação mais precisa dos periódicos científicos.
\end{abstract}

Palavras-chave: Fator de Impacto. Índice $h$. Indicadores bibliométricos. Periódicos da Ciência da Informação. Avaliação científica. 


\section{Introdução}

A avaliação da produção científica em diferentes áreas, instituições e países tornou-se um instrumento necessário para mapear a ciência e para subsidiar as tomadas de decisões em políticas científicas dos países. Neste cenário, os indicadores bibliométricos vêm ocupando um papel relevante no processo avaliativo da produção científica e, entre eles, destacam-se o Fator de Impacto (FI) e o Índice $h$.

O FI, criado em 1955 por Eugene Garfield para a avaliação de impacto de revistas e periódicos científicos (GARFIELD, 2005), pode ser entendido como o número médio de citações recebidas por trabalho em um determinado período. É definido como o número médio de citações recebidas por trabalhos de um determinado periódico científico em um determinado período. Desde então, firmou-se como um instrumento de avaliação de periódicos científicos, sendo atualmente calculado pelo The Clarivate Analytics Impact Factor, que define o FI de um periódico dividindo o número de citações pelo número de documentos publicados nos dois anos anteriores (CLARIVATE ANALYTICS, 2018). Embora o Clarivate Analytics seja o proprietário do indicador FI e também o responsável pela publicação do Journal Citation Report (JRC), outras bases de dados como a Scopus e SciELO fornecem informações para os pesquisadores calcularem o FI.

Hirsch (2005) desenvolveu um índice bibliométrico, denominado Índice $h$, que considera em sua estrutura aspectos relativos ao impacto científico (citação) e à produtividade (número de artigos produzidos) do pesquisador.

$\mathrm{O}$ fato de combinar produtividade e impacto científico configura uma característica relevante do índice $h$, que o leva a ser considerado um indicador eficaz por conjugar produção e impacto científico (MARQUES, 2013). Egghe (2010) ressaltou, ainda, que $h$ é uma medida robusta em dois aspectos, pois não é influenciada por um conjunto de poucas citações, nem por artigos altamente citados.

Esses indicadores foram construídos fundamentados nas bases de dados reconhecidas mundialmente, a saber: Web of Science (WoS) e Scopus. Durante 
muito tempo o Institute for Scientific Information (ISI) manteve o domínio de cobertura da literatura de diferentes áreas do conhecimento até surgir a base de dados Scopus. A Scopus tornou-se mundialmente reconhecida no mercado da literatura científica, incluindo publicações comerciais, periódicos científicos, relatórios, livros, conferências, materiais editoriais e revistas de acesso aberto (CHADEGANI et al., 2013). A produção científica indexada nessas grandes bases de dados é denominada ciência mainstream, aquela menos visível na ciência internacional é considerada ciência periférica (VELHO, 1985). A Scopus apresenta um maior número de periódicos brasileiros indexados em relação à base multidisciplinar WoS (MIGUEL, 2011; HERNÁNDEZ-GONZÁLEZ et al., 2016). O índice $h$ e o FI são indicadores muito utilizados na avaliação da produção científica indexada nessas bases de dados. Esses indicadores compõem o conjunto de métricas quantitativas usados como referências de dimensionamento da produção científica.

Em virtude da importância desses indicadores, a literatura apresenta alguns trabalhos que discutem o uso desses indicadores de forma conjunta ou o uso de indicadores compostos nas avaliações da produção científica de universidades, instituições e países. O estudo de Schubert e Glänzel (2007) demonstrou um modelo matemático para explicar a relação entre o índice $h$ e o FI. Vanclay (2008) mostrou de forma gráfica o relacionamento de entre o índice $h \mathrm{e}$ FI. Egghe et al. (2009) desenvolveu um modelo matemático que assume uma relação aproximadamente linear entre o índice $h$ e o FI. Bador e Lafouge (2009) identificou que a relação entre o índice $h$ e o FI é uma relação linear, combinou os valores desses indicadores utilizando a distribuição da média e desvio padrão e criou quatro grupos de classificação de periódicos da área de farmacologia e psiquiatria.

Nesse contexto, o presente trabalho tem por objetivo propor uma forma de avaliação de periódicos científicos, combinando o FI e o índice $h$, a fim de contribuir para a discussão da necessidade da utilização, em conjunto, de diferentes métricas científicas, que no processo avaliativo.

O presente artigo é uma versão ampliada do trabalho apresentado no $6^{\circ}$ Encontro Brasileiro de Bibliometria e Cientometria (6 $\left.6^{\circ} \mathrm{EBBC}\right)$, intitulado 
Avaliação de periódicos em Ciência da Informação: junção dos indicadores Fator de Impacto e Índice h (SILVA; ALMEIDA; GRACIO, 2018)

\section{Indicadores bibliométricos e cientométricos}

Em geral, os indicadores em Ciência e Tecnologia (C\&T) são medidas quantitativas usadas para expressar, quantificar ou representar conceitos relacionados ao desempenho, processo ou grau de desenvolvimento científico ou tecnológico de uma área do conhecimento. Nesse sentido, eles são necessários e relevantes para a análise e avaliação dos diferentes ramos da ciência (GRÁCIO, OLIVEIRA, 2012; JANNUZZI, 2002).

Santos e Kobashi (2005) consideram que os indicadores quantitativos não representam uma verdade absoluta sobre o estado da ciência e da tecnologia, mas são aproximações da realidade ou uma expressão incompleta dela. Apontam que a utilização desses indicadores deve ser comparativa para ter significado e aconselham que a confiança excessiva neles seja evitada. Os autores consideram a seleção e a construção de indicadores adequados aos diferentes contextos uma tarefa extremamente complexa. Devido a esse fato, parte dos esforços em Ciência, Tecnologia e Inovação concentram-se na elaboração de metodologias apropriadas para a formulação de indicadores adequados e representativos do universo analisado.

Narin (1994) relata que os indicadores quantitativos possibilitam o entendimento do desenvolvimento científico e tecnológico contemporâneo. Sugere que os indicadores podem ser classificados em três diferentes perspectivas: 1. indicadores de atividade; 2. indicadores de impacto e 3 . indicadores de ligação. Os indicadores de atividade mensuram a atividade e desenvolvimento da ciência, os de impacto mensuram o impacto das atividades de pesquisa, e os de ligação mensuram as ligações entre as organizações produtoras das atividades científicas e as ligações de conhecimento entre suas áreas ou temáticas (GREGOLIN et al., 2005; NARIN, 1994).

Considerando que o objeto de estudo deste trabalho está no escopo dos indicadores de citação, apresenta-se, na seção seguinte, conceitos e definições acerca do assunto. 


\subsection{Indicadores de citação}

Baseada no conjunto de referências arroladas no final de cada trabalho científico, a análise de citação constitui importante parte da Bibliometria. Segundo Araújo (2006), a análise de citação permite identificar características específicas da comunicação científica: os autores mais citados; a frente de pesquisa; a procedência geográfica e/ou institucional; a tipologia documental mais citada; a meia-vida da literatura usada; a obsolescência da literatura; o idioma predominante na literatura citada; o core de periódicos que compõem um campo.

Logo, a análise de citação tem por objetivo medir o impacto e a visibilidade de determinados autores, instituições, periódicos ou país dentro de uma comunidade científica e possibilita a análise das fontes de informações utilizadas. Apesar de haver críticas relacionadas à sua utilização, os estudos de citação têm demonstrado confiabilidade como instrumento de mensuração do impacto das publicações (GLÄNZEL, 2003; VANZ; CAREGNATO, 2003).

Algumas questões associadas ao uso de indicadores de citação referemse às diferenças epistemológicas e de práticas de comunicação científica entre as áreas do conhecimento; às motivações para a citação e para a não citação; à própria natureza da publicação (se é de revisão ou original); à barreira linguística; à origem da publicação (de países periféricos ou mainstream) (OLIVEIRA; GRÁCIO, 2011; VANZ; CAREGNATO, 2003).

Vinkler (2010) destacou que os indicadores cientométricos podem ser classificados de acordo com o número de conjuntos de dados utilizados e podem ser chamados de indicadores 1. simples, 2. compostos ou 3. complexos. Os indicadores simples utilizam um único aspecto dos sistemas cientométricos e são representados por uma única variável cientométrica e com um único nível hierárquico. Pode-se citar, como exemplo, o número total de citações de um conjunto de publicações. Os indicadores complexos utilizam dois ou mais conjuntos cientométricos ou consideram uma única variável cientométrica com mais de um nível hierárquico. Já os indicadores compostos são formados por vários indicadores simples ou complexos, de preferência com fatores de 
ponderação, e cada fator representa um aspecto especial de um dado sistema cientométrico. Eles consistem em índices parciais bem selecionados como, por exemplo, podem caracterizar a atividade global de organizações (Instituição ou país) dentro de um sistema correspondente (universidade, mundo) contendo várias organizações.

\subsection{1 Índice $h$}

O índice $h$ é um indicador bibliométrico destinado a medir, simultaneamente, o volume e o impacto da produção científica de um pesquisador. $\mathrm{O}$ desenvolvimento desse índice surgiu da indagação sobre como quantificar o impacto acumulado dos resultados das investigações de um pesquisador como reflexo da relevância científica da sua atuação (HIRSCH, 2005). Uma das justificativas apresentadas pelo autor para a proposição desse indicador foi o fato de os recursos serem limitados e a avaliação ser necessária.

$\mathrm{O}$ autor relatou que o registro do conjunto de publicações de um indivíduo e de suas respectivas citações configura um corpus de informação relevante sobre o desempenho e o reconhecimento junto à comunidade científica. Ele propôs, então, o índice $h$, como um número particularmente simples de ser obtido e útil para caracterizar o impacto científico de um pesquisador.

Hirsch (2005, p. 1, tradução nossa $)^{1}$ definiu o índice $h$ da seguinte forma: "Um cientista tem índice $\mathrm{h}$, se $\mathrm{h}$ de seus $\mathrm{N}_{\mathrm{p}}$ artigos têm ao menos $\mathrm{h}$ citações cada, e os outros $\left(\mathrm{N}_{\mathrm{p}}-\mathrm{h}\right)$ artigos têm não mais que h citações cada". Em que: $h$ = valor do índice $h$ e $N_{p}=$ número de publicações.

Egghe (2010, p. 3, tradução nossa) ${ }^{2}$ destacou a seguinte definição equivalente para o índice $h$ apresentada por Hirsch: "Ranquear os artigos de um autor e, em ordem decrescente, colocar o número de citações recebidas. O índice $h$ do autor é o maior valor $\mathrm{r}=h$ que o trabalho teve no rank $1,2, \ldots, \mathrm{h}$, tendo $h$ ou mais citações." 
Observa-se, assim, uma característica relevante do índice $h$ : ser uma medida capaz de combinar quantidade e qualidade da produção acadêmica e, por esse motivo, é considerado um indicador eficaz (EGGHE, 2010; MARQUES, 2013).

Desse modo, o índice $h$ identifica o núcleo mais produtivo das publicações científicas do avaliado em termos daqueles trabalhos que receberam maiores número de citações. Esse grupo de artigos é composto pelos primeiros documentos $h$ e é chamado de núcleo Hirsch ou $h$-core. Os documentos que compõem esse grupo têm alto impacto, no que diz respeito à carreira do cientista (BURRELL, 2007; JIN et al., 2007; ROUSSEAU, 2006).

O índice $h$ é considerado robusto, pois não é influenciado por um conjunto de artigos com poucas citações, nem por artigos altamente citados (EGGHE, 2010). Também há a possibilidade de utilizá-lo na tomada de decisões sobre promoções, alocação de verbas e atribuição de prêmios. Ele tem desempenho melhor do que o de outros indicadores bibliométricos utilizados para a avaliação da produtividade científica de um pesquisador (número de artigos, número de citações, citações por artigo e número de artigos altamente citados) quando utilizado de forma isolada (MARQUES, 2013).

\subsubsection{Fator de Impacto}

O FI foi criado em 1955, por Eugene Garfield, do ISI, com o propósito de selecionar revistas científicas da base de dados Science Citation Index (SCI), partindo da contagem de citação do autor, em uma revista pertencente a um núcleo de grupos grandes e de revistas altamente citadas que precisava ser coberto pelo SCI (GARFIELD, 1955; GARFIELD; SHER, 1963).

A forma de mensuração quantitativa do "Fator de Impacto" foi baseada em dois elementos: o numerador, que é o número de citação do ano corrente de quaisquer itens publicados nos dois anos anteriores, e o denominador, que é o número de artigos publicados nos mesmos dois anos (GARFIELD, 1999, 2005). Entretanto, a literatura científica apresenta várias críticas e limitações quanto a sua utilização (FERNANDES-LLIMÓS, 2003; GLÄNZEL; MOED, 2002; 
KALTENBORN, 2004; SEGLEN, 1997; SIMONS, 2008; WALTMAN, 2016; WALTMAN; TRAAG, 2017).

Atualmente, o FI é utilizado na avaliação do impacto de revistas e periódicos, gestão e política de pesquisa, recuperação de informações e na alocação de recursos, além de assumir um papel importante na avaliação da produção científica de grupos de pesquisa, universidades, institutos e países.

\section{Procedimentos metodológicos}

Para realização deste estudo optou-se pela Scopus, selecionando a base de dados no mecanismo de busca do portal SCImago Journal \& Country Rank, pois apresenta um maior número de periódicos brasileiros indexados em relação à base multidisciplinar WoS (MIGUEL, 2011). Em seguida, nas opções área "Social Sciences", subárea "Library and Information Sciences" e tipo de publicação "Journal", identificaram-se os periódicos indexados na base de dados Scopus nos anos 2012, 2013 e 2014.

Com esse critério, elegeram-se 195 periódicos da área de Ciências Sociais, especificamente consignados à Ciência da Informação. Em seguida, selecionaram-se apenas periódicos que continuavam indexados no período do estudo, totalizando 168 periódicos.

A seguir, para esse rol de periódicos, identificaram-se suas posições no ranking e seus respectivos quartis $\left(\mathrm{Q}_{1}, \mathrm{Q}_{2}, \mathrm{Q}_{3}\right.$ e $\left.\mathrm{Q}_{4}\right)$, para o ano de referência 2015. Ainda, para cada periódico foram levantadas as informações da base Scopus: índice $h$, total de documentos publicados em 2015, total de documentos dos últimos três anos e total de citações nos últimos três anos. Para cada um dos periódicos, indexados nos respectivos anos de 2012 a 2014, utilizando o software Excel, foi calculado o FI para o ano de referência 2015, considerando um período de três anos, a partir de cada base de dados (Scopus), conforme a fórmula:

$$
F I_{2015}=\frac{\text { citações }_{2012}+\text { citações }_{2013}+\text { citações }_{2014}}{\text { artigos publicados }_{2012}+\text { artigos publicados }_{2013}+\text { artigos publicados }_{2014}}
$$


Calcularam-se estatísticas descritivas do índice $h$ e FI. Em seguida, o coeficiente de correlação de Pearson e o gráfico de dispersão com o propósito de identificar o grau e intensidade do relacionamento dessas duas métricas. O coeficiente de correlação de Pearson resulta em um valor que pode variar dentro do intervalo de -1 a 1 , quanto mais próximo de -1 ou 1 indica correlação forte e o sentido pode ser negativo ou positivo. Embora, o índice $h$ e o FI apresentarem relação linear positiva forte, foram detectados valores atípicos (outliers) que influenciam o valor do cálculo do coeficiente de Pearson.

Dessa forma, optou-se por confrontar os dados conforme os quartis calculados para cada um dos indicadores: FI e índice $h$. Os Quartis Concordantes são compostos por aqueles periódicos que satisfizeram a seguinte condição: periódicos com valor de FI classificado no i-ésimo quartil e, simultaneamente, valor do índice $h$ classificado no $i$-ésimo quartil, para $1 \leq \mathrm{i} \leq 4$.

Complementarmente, foram considerados Quartis Discordantes aqueles em que os valores do FI e valores do índice $h$ não concordavam quanto ao quartil. Utilizando o software SPSS versão 21.0., foi aplicado o teste qui-quadrado, com nível de significância de 5\%, a fim de verificar a independência dos Quartis a que pertencem os 168 periódicos analisados.

A seguir foi realizada a análise de cluster, comumente chamada de “agrupamento do vizinho mais próximo" (COURTIAL, 1994; HE, 2008), com o objetivo de agrupar os periódicos discordantes quanto aos posicionamentos dos quartis relativos aos dois indicadores (FI e índice $h$ ) e verificar os agrupamentos entre eles no que se refere às similaridades relativas aos seus desempenhos nesses indicadores.

\section{Análise dos resultados}

\subsection{Correlação entre o FI e o índice $h$}

Inicialmente foi calculado o coeficiente de correlação de Pearson entre o FI e o índice $h$ para o conjunto de 168 periódicos, sendo o FI e o índice $h$ referentes ao ano de 2015, com o intuito de verificar a força da associação entre os dois indicadores bibliométricos para esse conjunto de informações. 
Verificou-se uma correlação forte entre o índice $h$ e FI $(0,70)$, indicando a existência de relação linear positiva entre essas duas medidas. Bador e Lafouge (2010) analisaram os indicadores FI e índice $h$ dos periódicos de farmacologia e psiquiatria e apontaram correlação linear forte e de sentido negativo, propuseram a criação de quatro grupos combinando os valores do FI e do índice h usando a distribuição das frequências relativas desses valores considerando as estimativas da média e o desvio padrão.

No presente estudo, apesar de a correlação de Pearson ser considerada uma relação linear forte e positiva, ao analisar o diagrama de dispersão, apresentado pelo Gráfico 1, identificaram-se alguns valores discrepantes para os dois indicadores.

Gráfico 1 - Diagrama de dispersão relativo ao índice $h$ e FI para os periódicos consignados à Ciência da Informação (2015)

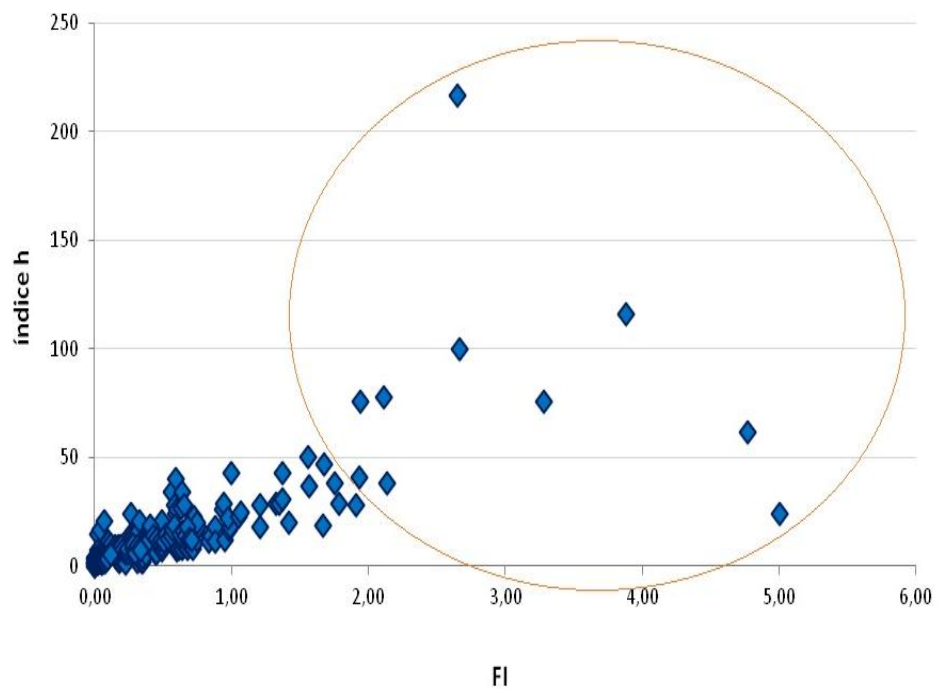

Fonte: Elaborado pelos autores (2018).

Observa-se por meio do Gráfico 1 que a maioria dos valores do índice $h \mathrm{e}$ FI estão concentrados no intervalo de valores de índice $h$ de 0 a 50 e valores de FI de 0 a 1 , no entanto, foram identificados alguns valores extremos para esses indicadores. Destaca-se os periódicos Bulletin des Bibliotheques de France $(h=$ 78; FI $=2,11)$, Archives $(h=38 ; \mathrm{FI}=2,14)$, Script and Print $(h=217 ; \mathrm{FI}=2,65)$, Library ( $h=100 ; \mathrm{FI}=2,66)$, Informacion, Cultura y Sociedad $(h=76 ; \mathrm{FI}=3,28)$, Prologue $(h=116 ; \mathrm{FI}=3,88)$, Notes and queries $(h=62 ; \mathrm{FI}=4,77)$, entre outros. No entanto, o coeficiente de correlação de Pearson é considerado uma medida 
estatística sensível, quando o conjunto de dados tem a presença de valores extremos.

Diante do exposto, optou-se em agrupar os valores do índice $h$ e FI de acordo com os seus respectivos quartis. $\mathrm{O}$ agrupamento resultou na combinação dos dois indicadores criando grupos de quartis.

A Tabela 1 apresenta a composição dos quadrantes concordantes (azuis) e discordantes (elementos fora da diagonal principal), em que se observa que aproximadamente $58 \%$ dos periódicos tiveram posicionamento similar quanto aos quartis do FI e do índice $h$. Por outro lado, 25\% dos periódicos alcançou posicionamento melhor em relação ao quartil do FI em comparação ao alcançado pelo posicionamento do índice $h$ e a menor porcentagem (17\%) foi de periódicos com desempenho inverso a este. A aplicação do teste qui-quadrado evidenciou que há associação estatisticamente significante entre os quartis do FI e do índice $h$ dos periódicos, com uma tendência de os periódicos serem classificados nos mesmos quartis segundo esses indicadores.

Tabela 1 - Distribuição dos 168 periódicos da Ciência da Informação, indexados na Scopus, segundo a composição dos quadrantes constituídos a partir da combinação entre FI e índice $h$

\begin{tabular}{cc|c|c|c|c|c}
\hline Indice $h$ & Fator de Impacto & $Q_{1}$ & $Q_{2}$ & $Q_{3}$ & $Q_{4}$ & Total \\
\hline$Q_{1}$ & 30 & 9 & 2 & 1 & $\mathbf{4 2}$ \\
\hline$Q_{2}$ & 12 & 17 & 8 & 3 & $\mathbf{4 0}$ \\
\hline$Q_{3}$ & 0 & 16 & 18 & 6 & $\mathbf{4 0}$ \\
\hline$Q_{4}$ & 0 & 0 & 14 & 32 & $\mathbf{4 6}$ \\
\hline \multicolumn{2}{r|}{ Total } & $\mathbf{4 2}$ & $\mathbf{4 2}$ & $\mathbf{4 2}$ & $\mathbf{4 2}$ & $\mathbf{1 6 8}$ \\
\hline
\end{tabular}

Fonte: Elaborada pelos autores (2018).

A Tabela 2 apresenta as estatísticas descritivas para o FI e o índice $h$ dos periódicos, em que se observa que, em média, o índice $h$ dos periódicos é igual a 16,62 e desvio padrão de 23,34, o que determinou um coeficiente de variação de 140\%, indicando uma dispersão altíssima em relação a este indicador. Ainda, há periódicos cujo índice $h$ é igual a zero, a saber: Information Communication and Society e o Journal of Cheminformatics.

Além disso, 25\% dos periódicos $\left(\mathrm{Q}_{4}\right)$ têm índice $h$ com valor menor ou igual a 5. A mediana dos periódicos é $h=10$. Por outro lado, 25\% ( $\left.\mathrm{Q}_{1}\right)$ dos periódicos têm índice $h$ maior que 20, todavia, com comportamento dos 

avaliação de periódicos científicos: uma aplicação no campo da Ciência da Informação

Deise Deolindo Silva, Cátia Cândida de Almeida e Maria Cláudia Grácio

periódicos bastante disperso (coeficiente de variação 83\%), assim como observado em $\mathrm{Q}_{4}$ (coeficiente de variação 55\%), em decorrência, do comportamento discrepante do periódico IEEE Transactionson Information Theory com índice $h$ com valor outlier (217).

\subsection{Estatísticas descritivas}

A seguir são apresentadas as estatísticas descritivas, por quartil e geral, para o FI e índice $h$ dos periódicos consignados à Ciência da Informação, obtidos por meio da base Scopus considerando o ano de 2015 para a análise.

Tabela 2 - Estatísticas descritivas para o FI e o índice $h$ dos periódicos consignados à Ciência da Informação, obtidos por meio da base Scopus para o ano de 2015

\begin{tabular}{|c|c|c|c|c|c|c|c|}
\hline & \multicolumn{7}{|c|}{ Estatística descritiva } \\
\hline & Métricas & Mínimo & Máximo & Mediana & Média & Desvio Padrão & Coef.Var. \\
\hline \multirow{2}{*}{ Geral } & FI-2015 & 0 & 5,00 & 0,387 & 0,623 & 0,787 & $126 \%$ \\
\hline & Índice $h-2015$ & 0 & 217 & 10 & 16,625 & 23,342 & $140 \%$ \\
\hline \multirow{2}{*}{$\mathrm{Q}_{4}$} & FI-2015 & 0 & 0,120 & 0,034 & 0,038 & 0,033 & $86 \%$ \\
\hline & Índice $h-2015$ & 0 & 5 & 2,5 & 2,437 & 1,342 & $55 \%$ \\
\hline \multirow{2}{*}{$\mathrm{Q}_{3}$} & FI-2015 & 0,151 & 0,333 & 0,235 & 0,233 & 0,063 & $27 \%$ \\
\hline & Índice $h-2015$ & 6 & 10 & 8 & 7,666 & 1,328 & $17 \%$ \\
\hline \multirow{2}{*}{$\mathrm{Q}_{2}$} & FI-2015 & 0,395 & 0,711 & 0,564 & 0,556 & 0,101 & $18 \%$ \\
\hline & Índice $h-2015$ & 11 & 19 & 14 & 14,529 & 2,502 & $17 \%$ \\
\hline \multirow{2}{*}{$\mathrm{Q}_{1}$} & FI-2015 & 0,727 & 5 & 1,565 & 1,870 & 1,094 & $58 \%$ \\
\hline & Índice $h-2015$ & 20 & 217 & 34 & 48,266 & 40,082 & $83 \%$ \\
\hline
\end{tabular}

Fonte: Elaborada pelos autores (2018).

O FI médio dos periódicos é 0,623, e altíssima dispersão (coeficiente de variação igual a 126\%). Identificaram-se sete periódicos com FI igual a zero, dado que o total de citação dos últimos três anos foi igual a zero, sendo eles: International Journal of Multimedia Information Retrieval, Transactions of the Cambridge Bibliographical Society, Harvard Library Bulletin, Microform and Digitization Review, Anales de Documentacion e Biblios.

Ainda, 25\% dos periódicos tem FI até 0,150, metade dos periódicos tem FI de até 0,387 e $25 \%$ dos periódicos tem FI maior que 0,725 . Nesse quartil (Q4), observou-se um comportamento discrepante de um periódico, Gazette dês Archives, com FI $=5$. 

avaliação de periódicos científicos: uma aplicação no campo da Ciência da Informação

Deise Deolindo Silva, Cátia Cândida de Almeida e Maria Cláudia Grácio

A Figura 1 apresenta o box-plot para FI e índice $h$, a fim de verificar a variabilidade em cada quartil, em que se observa que os box-plot's têm formatos similares para ambos os indicadores, apesar de eles terem escalas diferentes, o que evidencia similaridade na forma classificatória dos periódicos nos diferentes quartis.

Figura 1 - Box-plots para o FI e para o índice $h$ de acordo com os quartis dos periódicos consignados à Ciência da Informação, para o ano de 2015

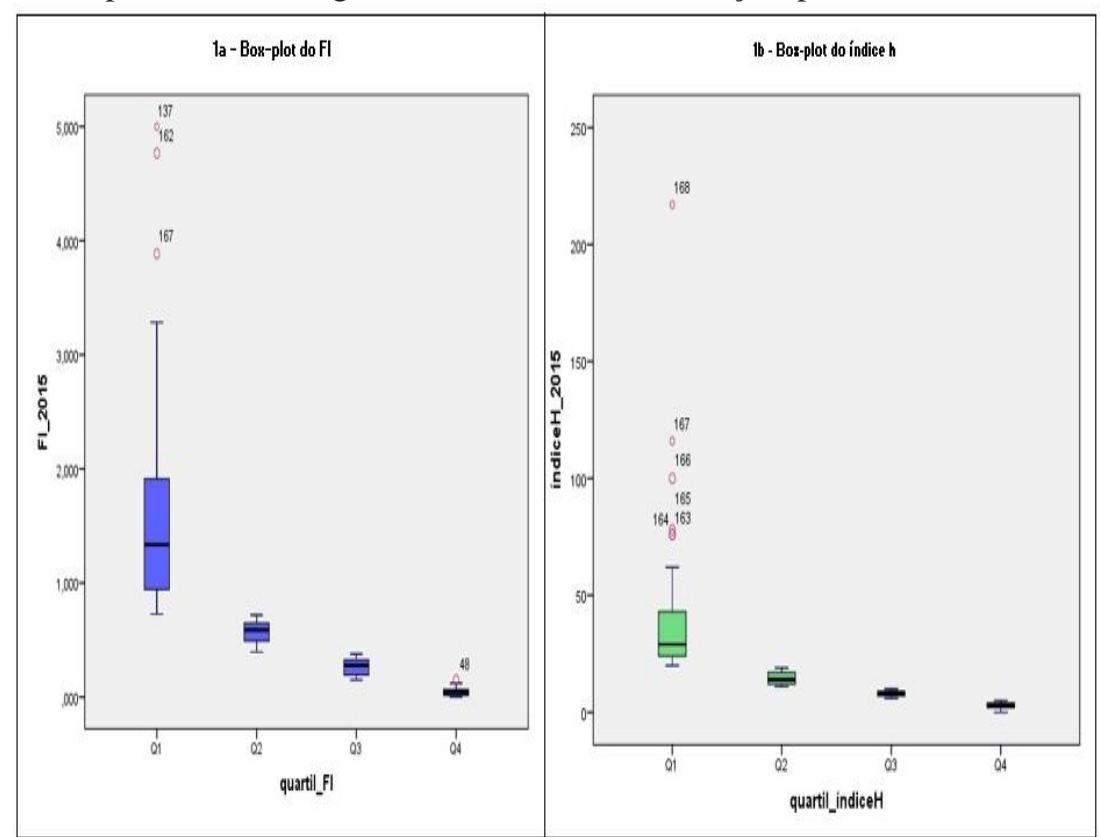

Fonte: Elaborada pelos autores (2018).

Identificou-se que para o quartil $\mathrm{Q}_{1}$, os valores dos FIs variam de 0,727 a 5,000, ao passo que os valores do índice $h$ variam de 19,75 a 217. Para o Quartil $\mathrm{Q}_{2}$, os valores dos FIs variam entre 0,396 a 0,719 e os valores do índice $h$ variam de 10 a 19,75. Para o quartil $Q_{3}$, os valores dos FIs variam de 0,152 a 0,379 e os valores do índice $h$ variam de 5 a 10 . Para o quartil $\mathrm{Q}_{4}$, os valores dos FIs variam entre 0 a 0,15 e os valores do Índice $h$ variam de 0 a 5 .

\subsection{Análise de cluster}

A análise de cluster para os 71 periódicos com posicionamentos discordantes (conforme Tabela 1) quanto aos quartis relativos ao FI e ao Índice $h$, determinou três agrupamentos, apresentados na Figura 2: Grupo 1: composto por 37 

avaliação de periódicos científicos: uma aplicação no campo da Ciência da Informação

Deise Deolindo Silva, Cátia Cândida de Almeida e Maria Cláudia Grácio

periódicos, com valores de FI de 0,000 a 0,500, com e índice $h$ de 0 a 20; Grupo 2: composto por 29 periódicos, com FI com valores entre 0,501 a 1,000 e índice $h$ entre 21 a 30; e Grupo 3: com cinco periódicos, com valores de FI acima de 1,000 e valores de índice $h$ de acima de 30 .

Figura 2 - Estrutura piramidal para os clusters dos periódicos consignados à Ciência da Informação com posicionamentos discordantes para o ano de 2015

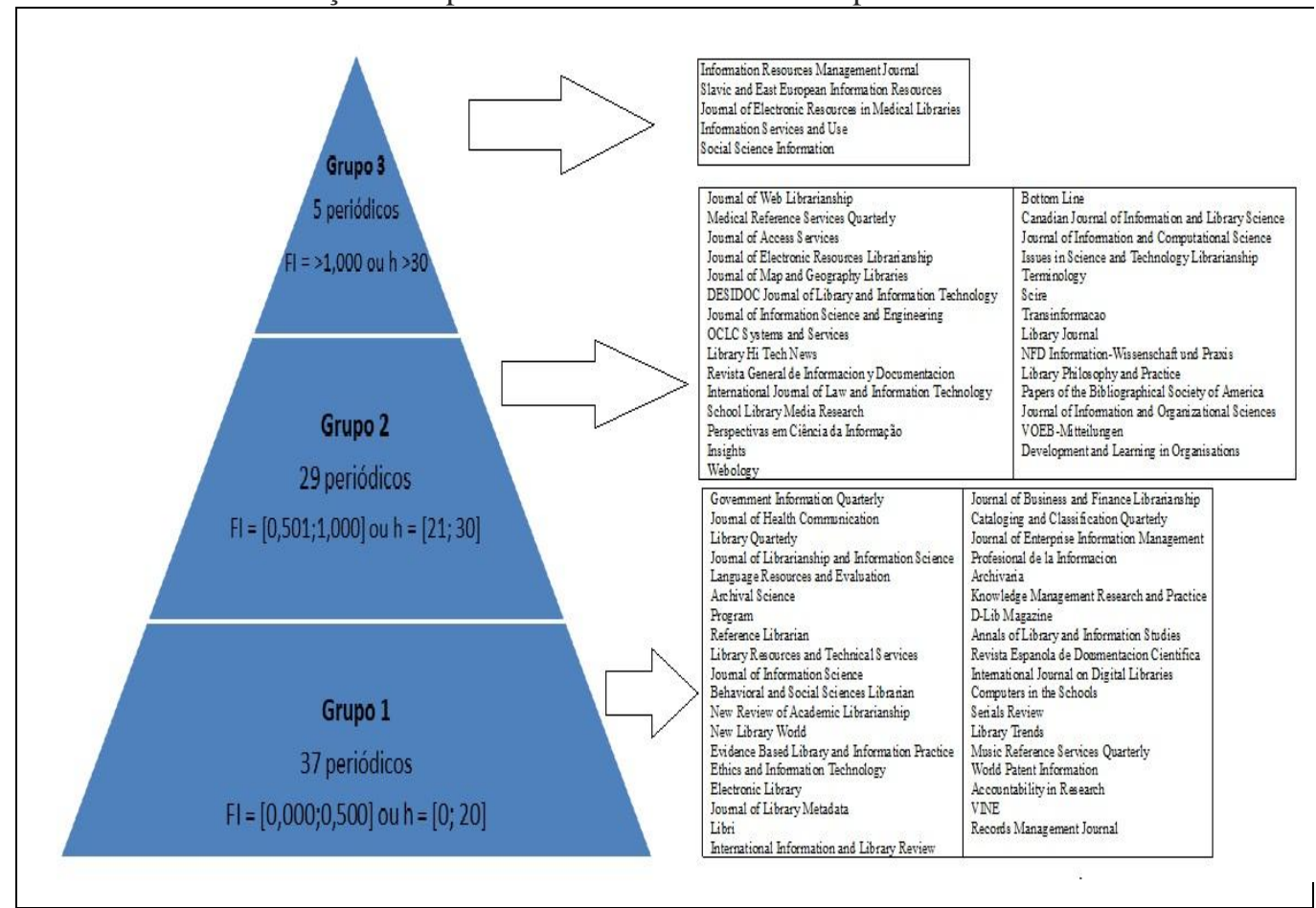

Fonte: Elaborada pelas autoras (2018).

Observa-se que o Grupo 3 agrupou os periódicos com FI maior 1,000 ou índice $h$ acima de 30, nesse grupo encontram-se cinco periódicos. Três com índice $h$ maior que 30 e com FI entre 0,501 e 1,000, a saber: Journal of Electronic Resources in Medical Libraries, Information Services and Use e Social Science Information e dois com FI maior que 1,000 e índice $h$ menor que 20: Information Resources Management Journal e Slavic and East European Information Resources.

O Grupo 2 reuniu periódicos com FI entre 0,501 e 1,000 ou com índice $h$ entre 21 e 30, classificaram-se 29 periódicos nesse agrupamento, dentre os quais 25 apresentaram FI > 0,501 e 9 com índice $h$ maior que 20. Para o Grupo 1, os periódicos agrupados têm FI até 0,500 ou índice $h$ até 20, identificaram-se 37 

avaliação de periódicos científicos: uma aplicação no campo da Ciência da Informação

Deise Deolindo Silva, Cátia Cândida de Almeida e Maria Cláudia Grácio

periódicos nesse grupo, dos quais quatro tem, simultaneamente, FI $\leq 0,120$ e índice $h \geq 10$.

A Figura 3 apresenta o diagrama de dispersão entre o FI e o índice $h$ para os periódicos discordantes, conforme a classificação obtida pela análise de cluster.

Figura 3 - Diagrama de dispersão para os periódicos consignados à Ciência da Informação discordantes, de acordo com a classificação do cluster

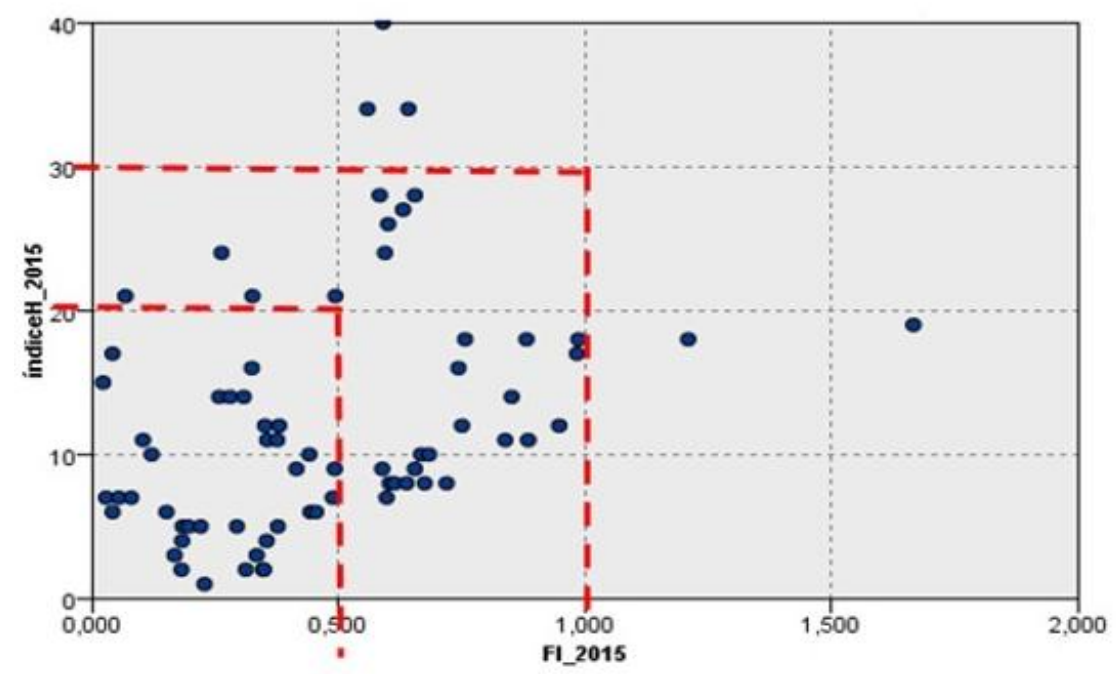

Fonte: Elaborada pelos autores (2018).

Percebe-se que ao utilizar essa metodologia conseguiu-se agrupar periódicos com características mais similares. O Grupo 1 - maior agrupamento - reuniu 52\% dos periódicos discordantes, os periódicos nele representados têm uma maior proximidade entre os valores do FI e índice $h$, determinando uma menor variabilidade. O Grupo 2 agrupou $41 \%$ dos periódicos discordantes, mas esse grupo caracteriza-se por reunir periódicos com FI e índices $h$ intermediários, no entanto, 69\% dos periódicos agrupados nesse grupo têm os valores de FI intermediários e índice $h$ menor que 20. O Grupo 3 - menor agrupamento - reuniu aqueles periódicos com maiores valores para os indicadores e observa-se maior distanciamento entre os periódicos presentes nele.

Deste modo, apesar de existir associação entre a classificação do índice $h$ e do FI nos mesmos quartis, evidenciados pelo teste qui-quadrado, ao nível de significância de 5\%; a análise de cluster contribuiu para formar grupos similares 
entre os periódicos discordantes e, assim, auxiliar a avaliação de periódicos em consignados em Ciência da Informação.

\section{Considerações finais}

Em função da intensa e atual utilização dos indicadores bibliométricos, em especial o FI e o Índice h, ambos destinados a representar o impacto científico dos artigos publicados, em análises e avaliações do desempenho científico, em nível micro, meso e macro, esta pesquisa buscou contribuir para o avanço dos estudos do tema, apresentando um procedimento de avaliação dos periódicos do campo da Ciência da Informação que considere simultaneamente esses dois indicadores.

Observou-se que, embora haja uma tendência de associação entre a classificação do Índice $h$ e do FI, em termos dos quartis das respectivas distribuições, para uma parcela significativa de periódicos as tendências são discordantes. Desse modo, evidenciou-se a necessidade da aplicação conjunta desses indicadores, considerando que representam perspectivas distintas do impacto do periódico na comunidade científica.

Nesse contexto, a análise articulada desses indicadores, por meio do método multivariado de cluster, permitiu evidenciar grupos de periódicos mais similares em uma visualização mais ampla.

Desse modo, conclui-se que a utilização conjunta dos indicadores FI e índice $h$ pode contribuir para uma avaliação mais ampla dos periódicos científicos, considerando que, embora a maioria tenha uma tendência de posicionamento similar quanto a esses indicadores, há uma parcela significativa de posicionamentos de quartis que são discordantes, levando a agrupamentos menores e mais similares entre si.

Finalizando, aponta-se a contribuição deste estudo para o conhecimento do comportamento dos indicadores bibliométricos, os quais têm sustentado políticas científicas e tomadas de decisão em diversos países. Sugere-se a realização de novos estudos que estendam a metodologia utilizada para outras áreas a fim de também se analisar as tendências de similaridade ou discrepância dos periódicos quanto ao FI e o Índice $h$. 

avaliação de periódicos científicos: uma aplicação no campo da Ciência da Informação

Deise Deolindo Silva, Cátia Cândida de Almeida e Maria Cláudia Grácio

\section{Referências}

ARAUJO, Carlos Alberto. Bibliometria: evolução histórica e questões atuais. Em Questão, v. 12, n. 1, p. 11-32, jan/jun, 2006.

BADOR, Pascal; LAFOUGE, Thierry. Comparative analysis between impact factor and h-index for pharmacology and psychiatry journals. Scientometrics, v. 84, n. 1, p.65-79, 2010.

BURRELL, Quentin L. On the h-index, the size of the Hirsch core and Jin's Aindex. Journal of Informetrics, v. 1, n. 2, p. 170-177, 2007.

CHADEGANI, Arezoo Aghaei; SALEHI, Hadi; YUNUS, Melor Md;

FARHADI, Hadi; FOOLADI, Masood; FARHADI, Maryam; EBRAHIM,

Nader Ale. Comparison between Two Main Academic Literature Collections:

Web of Science and Scopus Databases. Asian Social Science, Canada, v. 9, n. 5, 2013. Disponível em:

http://www.ccsenet.org/journal/index.php/ass/article/view/26960. Acesso em: 15 abr. 2017.

CLARIVATE ANALYTICS. The Clarivate Analytics Impact Factor. 2018. Disponível em: https://clarivate.com/essays/impact-factor/ . Acesso em: 05 jan. 2018.

COURTIAL, Jean-Paul. A co-word analysis of Scientometrics. Scientometrics, v.32, n. 3, p.251-260, 1994.

EGGHE, Leo.; LIANG, Liming; ROUSSEAU, Ronald. A Relational Between h-index and Impact Factor in the Power-Law Model. Journal of the American Society for Information Science and Technology, v. 60, n. 11, p. 2362-2365, 2009.

EGGHE, Leo. The Hirsch index and related impact measures. Annual review of information science and technology, v. 44, p. 65-114, 2010.

FERNANDÉZ-LLIMÓS, Fernando. Análisis de la cobertura del concepto de Pharmaceutical Care en fuentes primarias y secundarias de información. Granada: Universidad de Granada, 2003.

GARFIELD, Eugene. Citation indexes for science: A new dimension in documentation through association of ideas. Science, v.122, n. 3159, p. 108-11, 1955. Disponível em: http://garfield.library.upenn.edu/papers/science1955.pdf. Acesso em: 10 dez. 2017.

GARFIELD, Eugene; SHER, Irving H. New Factors in the Evaluation of Scientific Literature Through Citation Indexing. American Documentation, v. 4, n. 3, p. 195-201, 1963. Disponível em: http://garfield.library.upenn.edu/essays/v6p492y1983.pdf. Acesso em: 11 nov. 2017. 

avaliação de periódicos científicos: uma aplicação no campo da Ciência da Informação

Deise Deolindo Silva, Cátia Cândida de Almeida e Maria Cláudia Grácio

GARFIELD, Eugene. Journal impact factor: a brief review. Canadian Medical Association Journal, v. 161, n.8, p. 979-980, 1999. Disponível em: http://www.garfield.library.upenn.edu/papers/JournalimpactCMAJ1999.pdf . Acesso em: 20 nov. 2017.

GARFIELD, Eugene. The Agony and the Ecstasy - The History and Meaning of the Journal Impact. In: INTERNATIONAL CONGRESS ON PEER REVIEW AND BIOMEDICAL PUBLICATION, Chicago, 2005. Disponível em http://garfield.library.upenn.edu/papers/jifchicago2005.pdf. Acesso em 11 nov. 2017.

GLÄNZEL, Wolfgang; MOED, Floribert Henk. Journal impact measures in bibliometric research. Scientometrics, v. 53, n. 2, p. 171-193, 2002.

GLÄNZEL, Wolfgang. Bibliometrics as a research field: a course on theory and application of bibliometric indicators. [S.1.]: Coursehandouts, 2003.

GRÁCIO, Maria Cláudia Cabrini; OLIVEIRA, Ely Francina Tanuri. A inserção e o impacto internacional da pesquisa brasileira em "estudos métricos": uma análise na base Scopus. Tendências da Pesquisa Brasileira em Ciência da Informação, v. 5, n. 1, 2012.

GREGOLIN, José Ângelo Rodrigues. FARIA, Leandro Innocentini Lopes de; HOFFMANN, Wanda; QUONIAM, Luc.; QUEYRAS, Joachim. Análise da produção científica a partir de indicadores bibliométricos. In: Francisco Romeu Landi; Regina Gusmão. (Org.). Indicadores de Ciência, Tecnologia e Inovação em São Paulo 2004. 1. ed.São Paulo: Fapesp, 2005. p. 1-44. (v. 1).

HE, Qin. Knowledge discovery through co-word analysis. Library Trends, v.48, n. 3, p.133-159, 2008.

HERNÁNDEZ-GONZÁLEZ, V.; SANS-ROSELL, N. ; JOVÉ-DELTELL, M. C.; REVERTER-MASIA, J. Comparación entre web of science y scopus, estudio bibliométrico de las revistas de anatomía y morfología. Int. J. Morphol., v. 34, n. 4, p. 1369-1377, 2016.

HIRSCH, Jorge. E. An index to quantify an individual's scientific research output. Proceedings of the National Academy of Sciences of the United States of America, v. 102, n. 46, p. 16569-16572, Nov. 2005.

JANNUZZI, Paulo de Martino. Considerações sobre o uso, mau uso e abuso dos indicadores sociais na formulação e avaliação de políticas públicas municipais.

Revista de Administração Pública, v. 36, n. 1, p. 51-72, 2002.

KALTENBORN, Karl-Franz. Validity and fairness of the impact factor. German: Birkhäuser, 2004.

MARQUES, Fabrício. Os limites do índice-h: supervalorização do indicador que combina qualidade e quantidade da produção científica gera controvérsia.

Pesquisa Fapesp, n. 207, p. 35-39, 2013. 
MIGUEL, Sandra. Revistas y producción científica de América Latina y El Caribe: su visibilidad en SciELO, RedALyC y SCOPUS. Revista

Interamericana de Bibliotecología, v. 34, n. 2, p. 187-199, 2011.

NARIN, Francis. Patent bibliometrics. Scientometrics, v. 30, p. 147-155, 1994.

OLIVEIRA, Ely Francina Tanuri; GRÁCIO, Maria Cláudia Cabrini. Indicadores bibliométricos em ciência da informação: análise dos pesquisadores mais produtivos no tema estudo métricos na base Scopus. Perspectivas em Ciência da Informação, v. 16, n. 4, p. 16-28, out./dez. 2011.

ROUSSEAU, Ronald. New developments related to the Hirsch index. e-prints in library \& information science, 2006.

SANTOS, Raimundo Nonato Macedo; KOBASHI, Nair Yumiko. Aspectos metodológicos da produção de indicadores em ciência e tecnologia. In: ENCONTRO NACIONAL DE CIÊNCIA DA INFORMAÇÃO, 4, 2005. Anais... Salvador: UFBA, 2005.

SCHUBERT András; GLÄNZEL, Wolfgang. A systematic analysis of Hirschtype indices for journals. Journal of Informetrics, v. 1, p. 179-184, 2007.

SEGLEN, Per O. Citations and journal impact factors: Questionable indicators of research quality. Allergy, v. 52, n. 11, p. 1050-1056, 1997.

SILVA, Deise Deolindo; ALMEIDA, Cátia Cândida; GRACIO, Maria Cláudia Cabrini. Avaliação de periódicos em Ciência da Informação: junção dos indicadores Fator de Impacto e Índice h. In: ENCONTRO BRASILEIRO DE BIBLIOMETRIA E CIENTOMETRIA, 6., 2018, Rio de Janeiro. Anais... Rio de Janeiro, 2018. Disponível em:

https://ebbc.inf.br/ebbc6/docs/6EBBC2018v2018_07_27.pdf. Acesso em: 19 de dezembro de 2018.

SIMONS Kai. The misused Impact Factor. Science, v. 322, n. 10, 2008.

VANCLAY, Jerome K. Ranking forestry journals using the h-index. Journal of Informetrics, v. 2, p. 326-334, 2008.

VANZ, Samile Andréa de Souza; CAREGNATO, Sônia Elisa. Estudos de Citação: uma ferramenta para entender a comunicação. Em Questão, v. 9, n. 2, p. 295-307, jul./dez. 2003.

VELHO, Lea Maria Leme Strini. Como medir a Ciência? Revista Brasileira de Tecnologia, v. 16, n.1, p.35-41, jan./fev. 1985.

VINKLER Peter. The evaluation of research by scientometric indicators.

Chandos Publishing. Oxford Cambrigde, New Delhi, 2010. 
WALTMAN, Ludo. The importance of taking a clear position in the impact factor debate. Centre for Science and Technology Studies. Leiden University, Netherlands, 2016. Disponível em: https://www.cwts.n1/blog?article=n-q2w2c4. Acesso em: 06 dez. 2016.

WALTMAN, Ludo; TRAAG, Vincent. Use of the journal impact factor for assessing individual articles need not be wrong. Centre for Science and Technology Studies. Leiden University, Netherlands, 2017. Disponível em: https://www.cwts.n1/blog?article=n-q2w2c4. Acesso em: 03 ago. 2018.

\title{
Scientific evaluation of journals in Information Science: join of Impact Factor and $h$ index indicators
}

\begin{abstract}
Bibliometric indicators are a relevant subsidy to the assessment process of scientific production, especially at the meso and macro level, and to decision making in scientific policies. Among them, we highlight Impact Factor and h-index. Created for the impact assessment of scientific journals, Impact Factor is defined as the citation average reached by a scientific journal in a given period. The h-index considers in its formulation aspects related to the scientific impact (citation) and productivity (number of articles produced) of a researcher. Given the contemporary importance of these indicators, this research aims to propose a evaluate procedure to scientific journals that combines Impact Factor and h-index, in order to contribute to the discussion of the need for a multidimensional evaluation in the assessment processes. The values of Impact Factor and h-index of the 168 scientific periodicals from Information Science field that are indexed in the Scopus database for years 2012, 2013, 2014 were retrieved. Among the results, it was observed that, although there is a tendency of similar positioning regarding these indicators, for a significant portion of journals the trends are discordant. It is concluded that the combined use of Impact Factor and h-index contributes to a more precise evaluation of scientific journals.
\end{abstract}

Keywords: Impact Factor. h-index. Bibliometric indicators. Journals in Information Science. Scientific evaluation.

Recebido: 06/09/2018

Aceito: 03/12/2018

1 "A scientist has index $\mathrm{h}$ if $\mathrm{h}$ of his/her $\mathrm{Np}$ papers have at least $\mathrm{h}$ citations each, and the other $(\mathrm{Np}-\mathrm{h})$ papers have no more than $\mathrm{h}$ citations each".

2 If we rank the papers of an author in decreasing order of the number of citations they received then this author's $\mathrm{h}$-index is the highest rank $\mathrm{r}=\mathrm{h}$ such that the papers on ranks $1,2, \ldots, \mathrm{h}$ each have $\mathrm{h}$ or more citations. 